IJMMS 25:12 (2001) 811-817

PII. S016117120100535X

http://ijmms.hindawi.com

(c) Hindawi Publishing Corp.

\title{
ON THE APPLICATION OF ERGODIC THEORY TO ALTERNATING ENGEL SERIES
}

\author{
C. GANATSIOU
}

(Received 30 May 2000)

\begin{abstract}
We investigate the ergodic behaviour of the basic operator which generates the modified Engel-type alternating series representations of any number in $(0,1]$ in terms of rationals.
\end{abstract}

2000 Mathematics Subject Classification. Primary 11K55, 37A10, 37A25, 37A50, 60G10, 60J10, 60K99.

1. Introduction. Let $x$ be any number in the interval $I:=(0,1]$. Then according to a general alternating series algorithm introduced by A. Knopfmacher and J. Knopfmacher (see [5, 6]) $x$ has a unique finite or infinite representation in terms of a general alternating series of rationals as follows.

Let $T:(0,1] \rightarrow[0,1)$ be the transformation defined by

$$
\begin{gathered}
T(0)=0, \\
T(x)=\left(\frac{1}{[1 / x]}-x\right)\left(\left[\frac{1}{x}\right]+1\right), \text { for any } x \neq 0 .
\end{gathered}
$$

If $a_{1}=a_{1}(x)=[1 / x]$ and $a_{n+1}=a_{n+1}(x)=\left[1 /\left(T^{n}(x)\right)\right]$, for $T^{n}(x) \neq 0, n \in \mathrm{N}^{*}$, where $\mathrm{N}^{*}=\{1,2, \ldots\}$, we conclude that the transformation $T$ generates the following unique finite or infinite representation for every $x \in(0,1]$ :

$$
x=\frac{1}{a_{1}}+\sum_{n \geq 2} \frac{(-1)^{n-1}}{\left(a_{1}+1\right)\left(a_{2}+1\right) \cdots\left(a_{n-1}+1\right) a_{n}},
$$

where $\alpha_{1} \geq 1, \alpha_{n+1} \geq \alpha_{n}$, for any $n \in \mathrm{N}^{*}$.

Representation (1.3) is called "modified Engel-type" alternating expansion, while the positive integers $\alpha_{0}, \alpha_{1}, \ldots, \alpha_{n}$ are called digits of the above-mentioned expansion. In particular, we may say that in the case $T$ maps irrational numbers $x$ onto irrational numbers, the series is infinite. In fact $x$ is rational if the digits $\alpha_{i}$ satisfy the condition $\alpha_{i}=\alpha>1$, for $i \geq n+1$.

The purpose of the present paper is to give some important results arising from the application of ergodic theory to the alternating Engel-type series. More specific, we prove that the transformation $T$ related to the alternating Engel-type series is ergodic with respect to Lebesgue measure $\lambda$, but not $\lambda$-measure preserving.

For ordinary positive Engel series, an analogous problem has been previously investigated by F. Schweiger [9] (see also [2]). In particular, Schweiger found out that though 
the transformation related to the positive Engel series is ergodic, there is no measure which is preserved by this transformation and which is absolutely continuous with respect to Lebesgue measure.

The paper is organized as follows. In Section 2, we present some auxiliary results concerning the alternating Engel-type series in order to make more comprehensible the presentation of this paper. Section 3 includes the ergodic behaviour of the transformation $T$ generating the modified Engel-type expansions (1.3). Finally, in Section 4 we give a brief account of certain concepts of ergodic theory that we need throughout the paper.

2. Auxiliary results. We define by

$$
I_{n}=I_{n}\left(k_{1}, k_{2}, \ldots, k_{n}\right)=\left\{x \in(0,1] / \alpha_{1}(x)=k_{1}, \alpha_{2}(x)=k_{2}, \ldots, \alpha_{n}(x)=k_{n}\right\},
$$

for any $k_{1}, \ldots, k_{n} \in \mathrm{N}^{*}$, the set of all $x \in(0,1]$ which have a unique expansion of the form (1.3) such that the digits $\alpha_{1}(x), \ldots, \alpha_{n}(x)$ have the concrete values $k_{1}, \ldots, k_{n}$. Here $I_{0}=(0,1]$.

Then we obtain the following statement.

THEOREM 2.1. The set $I_{n}=I_{n}\left(k_{1}, k_{2}, \ldots, k_{n}\right)$ is bounded and its corresponding bounds are given by the relations

$$
\begin{aligned}
M_{n} \equiv & \sup I_{n}\left(k_{1}, k_{2}, \ldots, k_{n}\right) \\
= & \frac{1}{k_{1}}-\frac{1}{\left(k_{1}+1\right)} \frac{1}{k_{2}}+\cdots+(-1)^{n-1} \frac{1}{\left(k_{1}+1\right)\left(k_{2}+1\right) \cdots\left(k_{n-1}+1\right)} \frac{1}{k_{n}}, \\
m_{n} \equiv & \inf I_{n}\left(k_{1}, k_{2}, \ldots, k_{n}\right) \\
= & \frac{1}{k_{1}}-\frac{1}{\left(k_{1}+1\right)} \frac{1}{k_{2}}+\cdots+(-1)^{n-1} \frac{1}{\left(k_{1}+1\right)\left(k_{2}+1\right) \cdots\left(k_{n-1}+1\right)} \frac{1}{k_{n}} \\
& +(-1)^{n} \frac{1}{\left(k_{1}+1\right)\left(k_{2}+1\right) \cdots\left(k_{n}+1\right)},
\end{aligned}
$$

where $n$ is odd. If $n$ is an even number, then the above relations are inverted. Moreover, its Lebesgue measure is given by

$$
\lambda\left(I_{n}\right)=\prod_{j=1}^{n} \frac{1}{k_{j}+1},
$$

for any $\eta \in \mathrm{N}^{*}$.

For the proof of Theorem 2.1 we refer the reader to C. Ganatsiou [3]. Consider the set

$$
I_{r_{j-1}(x)}=\left\{x \in(0,1] / r_{j-1}(x)=\frac{1}{k_{j}}-\frac{1}{k_{j}+1} \cdot r_{j}(x), r_{j}(x) \in[0,1], k_{j} \geq 1, j \in \mathrm{N}^{*}\right\},
$$

where $r_{j}(x)$ is the $j$ th rest of the modified Engel-type alternating series (1.3) defined by

$$
r_{j}(x)=\frac{1}{\alpha_{j+1}(x)}-\frac{1}{\alpha_{j+1}(x)+1} \cdot r_{j+1}(x), \quad j=0,1,2, \ldots
$$


Then under the consideration of the probability space $\left((0,1], B_{(0,1]}, \lambda\right)$, where $\lambda$ is the Lebesgue measure we have the following theorem.

THEOREM 2.2. The rests $r_{n}(x), n=0,1,2, \ldots$ of the modified Engel-type alternating expansion satisfy the following relation:

$$
\begin{aligned}
\lambda\left(x \in(0,1] / r_{0}(x) \in I_{r_{0}(x)}, r_{1}(x) \in I_{r_{1}(x)}, \ldots, r_{n-1}(x) \in I_{r_{n-1}(x)}\right) & \\
& =\prod_{j=1}^{n} \lambda\left(x \in(0,1] / r_{j-1(x)} \in I_{r_{j-1}(x)}\right),
\end{aligned}
$$

where

$$
\lambda\left(x \in(0,1] / r_{j-1}(x) \in I_{r_{j-1}(x)}\right)=\frac{1}{k_{j}+1}, \quad j=1,2, \ldots
$$

PROoF. At first we prove the relation (2.7) for an arbitrary $j=1,2, \ldots$. If $\alpha_{j}(x)=k_{j}$, then by (2.5) we take that

$$
r_{j-1}(x)=\frac{1}{k_{j}}-\frac{1}{k_{j}+1} \cdot r_{j}(x), \quad r_{j}(x) \in[0,1], j \in \mathrm{N}^{*} .
$$

So if $r_{j}(x)=0$, then

$$
r_{j-1}(x)=\frac{1}{k_{j}}
$$

Equivalently we obtain that

$$
\sup I_{r_{j-1}(x)}=\frac{1}{k_{j}} .
$$

While if $r_{j}(x)=1$, then

$$
r_{j-1}(x)=\frac{1}{k_{j}\left(k_{j}+1\right)} .
$$

So

$$
\inf I_{r_{j-1}(x)}=\frac{1}{k_{j}\left(k_{j}+1\right)}
$$

Therefore, we get

$$
\lambda\left(x \in(0,1] / r_{j-1}(x) \in I_{r_{j-1}(x)}\right)=\frac{1}{k_{j}+1}, \quad j=1,2, \ldots
$$

In order to prove (2.6) we take the following cases. If 
(i) $n$ is odd, then

$$
\begin{aligned}
& \lambda(x \in\left.(0,1] / r_{0}(x) \in I_{r_{0}(x)}, r_{1}(x) \in I_{r_{1}(x)}, \ldots, r_{n-1}(x) \in I_{r_{n-1}(x)}\right) \\
&=\lambda\left(x \in(0,1] / r_{0}(x)=\frac{1}{k_{1}}-\frac{1}{k_{1}+1} \cdot r_{1}(x), r_{1}(x)=\frac{1}{k_{2}}-\frac{1}{\left(k_{2}+1\right)} \cdot r_{2}(x), \ldots,\right. \\
&\left.\quad r_{n-1}(x)=\frac{1}{k_{n}}-\frac{1}{\left(k_{n}+1\right)} \cdot r_{n}(x), r_{n}(x) \in[0,1]\right) \\
&=\lambda\left(x \in(0,1] / r_{0}(x)=\frac{1}{k_{1}}-\frac{1}{\left(k_{1}+1\right)} \frac{1}{k_{2}}+\cdots+(-1)^{n-1}\right. \\
& \quad \times \frac{1}{\left(k_{1}+1\right)\left(k_{2}+1\right) \cdots\left(k_{n-1}+1\right)} \frac{1}{k_{n}} \\
&\left.\quad+(-1)^{n} \frac{1}{\left(k_{1}+1\right)\left(k_{2}+1\right) \cdots\left(k_{n}+1\right)} r_{n}(x), r_{n}(x) \in[0,1]\right) \\
&=\lambda\left(I_{n}\left(k_{1}, k_{2}, \ldots, k_{n}\right)\right)=M_{n}-m_{n}=\prod_{j=1}^{n} \frac{1}{k_{j}+1}=\prod_{j=1}^{n} \lambda\left(x \in(0,1] / r_{j-1}(x) \in I_{r_{j-1}(x)}\right) .
\end{aligned}
$$

We repeat the same argument if

(ii) $n$ is an even number and the proof is complete.

This theorem corrects the escape of [3, Theorem 2.2.1].

3. The ergodic behaviour of the basic operator. Using the above auxiliary results and the definition (1.2) of the basic operator $T$, we are able to prove the following statement.

THEOREM 3.1. The modified Engel-type operator $T:(0,1] \rightarrow[0,1)$ is ergodic with respect to Lebesgue measure $\lambda$.

Proof. Define a linear map $\varphi_{n}=\varphi_{n}\left(k_{1}, k_{2}, \ldots, k_{n}\right):(0,1] \rightarrow I_{n}$ by

$$
\begin{aligned}
\phi_{n}(w) & =\sum_{j=1}^{n} \frac{(-1)^{j-1} \cdot \lambda\left(I_{j-1}\right)}{k_{j}}+(-1)^{n} w \lambda\left(I_{n}\right) \\
& =\sum_{j=1}^{n} \frac{(-1)^{j-1}}{\left(k_{1}+1\right) \cdots\left(k_{j-1}+1\right)} \cdot \frac{1}{k_{j}}+(-1)^{n} w \prod_{j=1}^{n} \frac{1}{k_{j}+1} .
\end{aligned}
$$

If $x \in I_{n}$, we have that

$$
\begin{aligned}
x & =\sum_{j=1}^{\infty} \frac{(-1)^{j-1}}{\left(\alpha_{1}+1\right) \cdots\left(\alpha_{j-1}+1\right)} \cdot \frac{1}{\alpha_{j}} \\
& =\sum_{j=1}^{n} \frac{(-1)^{j-1} \lambda\left(I_{j-1}\right)}{k_{j}}+\lambda\left(I_{n}\right) \sum_{j=n+1}^{\infty} \frac{(-1)^{j-1}}{\left(\alpha_{n+1}+1\right) \cdots\left(\alpha_{j-1}+1\right)} \cdot \frac{1}{\alpha_{j}} \\
& =\phi_{n}\left(T^{n}(x)\right) .
\end{aligned}
$$

Thus $\phi_{n}^{-1}=T^{n}: I_{n} \rightarrow I$. 
Moreover,

$$
M_{n}=\varphi_{n}(0), \quad m_{n}=\varphi_{n}(1), \quad \text { for any } n=1,3, \ldots
$$

with

$$
\begin{aligned}
\phi_{n}(0) & =\frac{1}{k_{1}}-\frac{1}{\left(k_{1}+1\right)} \cdot \frac{1}{k_{2}}+\cdots+(-1)^{n-1} \cdot \frac{1}{\left(k_{1}+1\right) \cdots\left(k_{n-1}+1\right)} \cdot \frac{1}{k_{n}} \\
& =\sum_{j=1}^{n} \frac{(-1)^{j-1} \cdot \lambda\left(I_{j-1}\right)}{k_{j}}, \\
\phi_{n}(1) & =\frac{1}{k_{j}}-\frac{1}{\left(k_{1}+1\right)} \cdot \frac{1}{k_{2}}+\cdots+(-1)^{n} \cdot \frac{1}{\left(k_{1}+1\right) \cdots\left(k_{n}+1\right)} \\
& =\sum_{j=1}^{n} \frac{(-1)^{j-1} \cdot \lambda\left(I_{j-1}\right)}{k_{j}}+(-1)^{n} \cdot \lambda\left(I_{n}\right) .
\end{aligned}
$$

(If $n$ is even then the above relations are inverted.)

Then for any interval $(a, b] \subseteq I$, we have

$$
\begin{aligned}
\lambda\left(T^{-n}(\alpha, b] \cap I_{n}\right) & =\lambda\left(\varphi_{n}(\alpha, b] \cap I_{n}\right)=\left|\varphi_{n}(b)-\varphi_{n}(\alpha)\right| \\
& =(b-\alpha) \cdot \lambda\left(I_{n}\right)=\lambda(a, b] \cdot \lambda\left(I_{n}\right) .
\end{aligned}
$$

Therefore

$$
\lambda\left(T^{-n} E \cap I_{n}\right)=\lambda(E) \cdot \lambda\left(I_{n}\right),
$$

for any set $E$ in the Boolean ring $R$ of all finite disjoint unions of intervals $(a, b] \subseteq I$. By using standard measure theory as in [4], it follows that the same equation holds for any Borel set $E$ in $I$.

Now let $E$ be a Borel set in $I$ such that $T^{-1} E=E$, where $T$ is a measurable, nonsingular transformation. Then we obtain that $T^{-n} E=E$, for any $n \geq 1$.

By relation (3.6) we take that

$$
\lambda\left(E \cap I_{n}\right)=\lambda(E) \cdot \lambda\left(I_{n}\right)
$$

or

$$
\lambda\left(E \cap I_{n}\right)=c \cdot \lambda\left(I_{n}\right), \quad \text { with } c=\lambda(E)>0 .
$$

If $U$ is the collection of all cylinders $I_{n}, n \geq 1$ and $\alpha_{j+1} \geq \alpha_{j}, \alpha_{1} \geq 1$, for any $j \geq 1$, then any open subinterval of $(0,1]$ is an at most denumerable (or countable) disjoint union of elements of $U(\lambda$ a.s.). Therefore

$$
\lambda(E \cap B)=c \cdot \lambda(B), \quad \text { with } c=\lambda(E)>0,
$$

for any set $B$ which is a countable disjoint union of the fundamental intervals $I_{n}$. Thus, by using Theorem 4.5, Definition 4.3, and the formula (3.9), the proof is complete.

REMARK 3.2. Even though the measurable, non-singular transformation $T$ defined by (1.2) is ergodic, it is not a $\lambda$-measure preserving transformation, since for any Borel 
set $E$ we have that

$$
\begin{aligned}
\lambda\left(T^{-1} E\right) & =\lambda\left(T^{-1} E \cap \bigcup_{m=1}^{\infty} I_{1}(m)\right)=\sum_{m=1}^{\infty} \lambda\left(T^{-1} E \cap I_{1}(m)\right) \\
& =\lambda(E) \sum_{m=1}^{\infty} \frac{1}{m+1} \neq \lambda(E),
\end{aligned}
$$

since the series $\sum_{m=1}^{\infty}(1 /(m+1))$ diverges.

An important property of the ergodic but not $\lambda$-measure preserving modified Engeltype transformation is the following form of Birkhoff-Riesz theorem (the so called Ergodic theorem) due to Dunford and Miller [1].

THEOREM 3.3. Let $T$ be the measurable, non-singular transformation of $((0,1]$, $\left.B_{(0,1]}, \lambda\right)$ into itself. Assume that there is a constant $M>0$ such that for any Borel set $E$,

$$
n^{-1} \sum_{k=0}^{n-1} \lambda\left(T^{-k} E\right) \leq M \lambda(E), \quad n \geq 1 .
$$

Then the a.s. limit

$$
\lim _{n \rightarrow \infty} n^{-1} \sum_{k=0}^{\infty} g\left(T^{k}(x)\right)
$$

for any integrable function $g$ on $I$, exists.

This means that many asymptotic results (such that the asymptotic frequency of the digits, the arithmetic and geometric means etc.) exists and they can be studied by using concepts and ideas of a specific theory.

\section{Appendix}

DeFINITION 4.1. Let $\left(\Omega_{1}, \mathscr{T}_{1}, P_{1}\right)$ and $\left(\Omega_{2}, \mathscr{T}_{2}, P_{2}\right)$ be two probability spaces.

(i) $A$ map $T: \Omega_{1} \rightarrow \Omega_{2}$ is called a transformation.

(ii) A transformation $T: \Omega_{1} \rightarrow \Omega_{2}$ is said to be measurable if for every $E \in \mathscr{T}_{2}$, $T^{-1} E \in \mathscr{T}_{1}$.

(iii) A measurable transformation $T: \Omega_{1} \rightarrow \Omega_{2}$ is said to be non-singular if for every $E \in \mathscr{T}_{2}$ with $P_{2}(E)=0, P_{1}\left(T^{-1} E\right)=0$.

DEFINITION 4.2. (i) A transformation $T: \Omega_{1} \rightarrow \Omega_{2}$ is called measure-preserving (or a homomorphism) if it is measurable and for any $E \in \mathscr{T}_{2}, P_{2}(E)=P_{1}\left(T^{-1} E\right)$.

(ii) A homomorphism is called an isomorphism if $T$ is an one-to-one map of $\Omega_{1}$ onto $\Omega_{2}$ and if $T^{-1}$ is also a homomorphism.

Now let the two above-mentioned probability spaces be identical.

DEFINITION 4.3. A measurable nonsingular transformation $T$ is called ergodic if the relation $T^{-1} E=E$, for $E \in \mathcal{T}$ implies $P(E)=0$ or $P(E)=1$. If the weaker assumption $T^{-1} E \subset E$, for $E \in \mathcal{T}$ already implies $P(E)=0$ or 1 , then $T$ is called strongly ergodic.

DEFINITION 4.4. If $P_{1}$ and $P_{2}$ are two probability measures on the probability space $(\Omega, \mathscr{T})$, then $P_{1}$ is said to be absolutely continuous with respect to $P_{2}$ if $P_{2}(E)=0$ 
implies $P_{1}(E)=0$ which we denote by $P_{1} \ll P_{2}$. In the case that $P_{1} \ll P_{2} \ll P_{1}$, then $P_{1}$ and $P_{2}$ are said to be equivalent.

Under the above definitions we take the following useful classical criterion for ergodicity of Knopp [7].

THEOREM 4.5. Let $E$ be a Lebesgue-measurable subset of $(0,1)$ with $P(E)>0$. Assume that there is a collection $J$ of subintervals of $(0,1)$ with the following properties:

(i) every open subinterval of $(0,1)$ is at most a denumerable union of disjoint elements of $J(P$ a.s. $)$ and

(ii) for every $B \in J, P(E B) \geq c P(B)$ with a constant $c>0$.

Then $P(E)=1$.

An important property of measure-preserving, ergodic transformations is the following Birkhoff-Riesz theorem (for the proof, see Riesz [8]).

THEOREM 4.6. Let $T$ be a measure-preserving, ergodic transformation of the probability space $(\Omega, \mathcal{T}, P)$. Let $f(x)(x \in \Omega)$ be integrable. Then a.s.

$$
\lim _{n \rightarrow \infty} n^{-1} \sum_{k=0}^{n-1} g\left(T^{k}(x)\right)=\int_{\Omega} g d P .
$$

ACKNOWLEDGEMENT. I am indebted to the referee for his valuable comments, which led to significant change of the first version.

\section{REFERENCES}

[1] N. Dunford and D. S. Miller, On the ergodic theorem, Trans. Amer. Math. Soc. 60 (1946), 538-549. MR 8,280b. Zbl 063.01187.

[2] J. Galambos, Representations of Real Numbers by Infinite Series, vol. 502, Springer-Verlag, Berlin, 1976, Lecture Notes in Mathematics. MR 58\#27873. Zbl 322.10002.

[3] C. Ganatsiou, On the stochastic behaviour of the digits in the modified Engel-type altenating series representations for real numbers, Proceedings of the Workshop on Differential Geometry, Global Analysis, and Lie Algebras (Aristotle University of Thessaloniki), Balkan Press, 1997.

[4] H. Jager and C. de Vroedt, Lüroth series and their ergodic properties, Nederl. Akad. Wetensch. Proc. Ser. A 31 (1969), 31-42. MR 39\#157. Zbl 167.32201.

[5] A. Knopfmacher and J. Knopfmacher, Two constructions of the real numbers via alternating series, Int. J. Math. Math. Sci. 12 (1989), no. 3, 603-613. MR 90k:26003b. Zbl 683.10008.

[6] _ New series and product representations for real numbers, manuscript, 1990.

[7] K. Knopp, Mengentheorestische Behandlung einiger probleme der diophantischen approximation und der transfiniten wahrscheinlichkeiten, Math. Ann. 95 (1926), 409-426 (German).

[8] F. Riesz, Sur la théorie ergodique, Comment. Math. Helv. 17 (1945), 221-239. MR 7,255b. Zbl 063.06500.

[9] F. Schweiger, Ergodische Theorie der Engelschen und Sylvesterschen Reihen, Czechoslovak Math. J. 20(95) (1970), 243-245. MR 41\#3712. Zbl 197.34501.

C. Ganatsiou: University of Thessalia, School of Technological Sciences, DepartMENT OF CiVIL ENGineERING, SeCtion of MATHEMATICS, Volos, GREeCE

E-mail address: ganatsio@tei 1ar.gr 


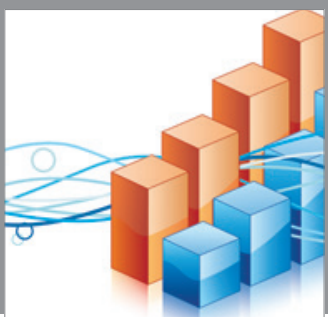

Advances in

Operations Research

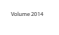

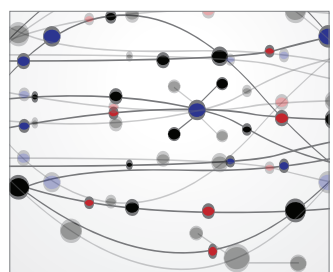

\section{The Scientific} World Journal
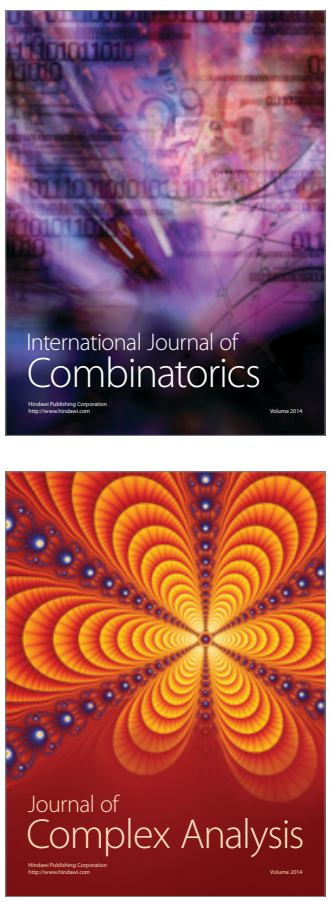

International Journal of

Mathematics and

Mathematical

Sciences
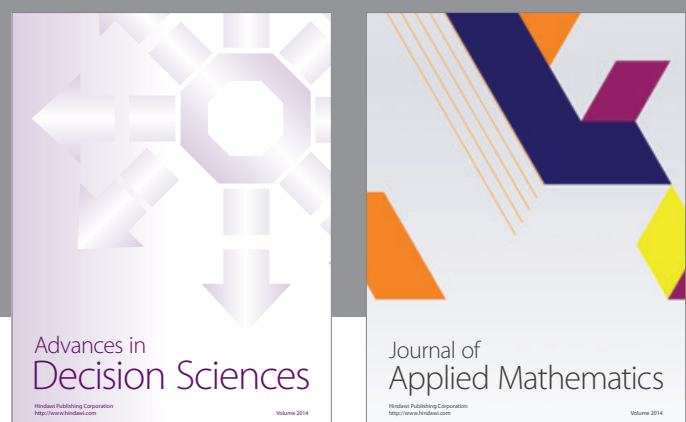

Journal of

Applied Mathematics
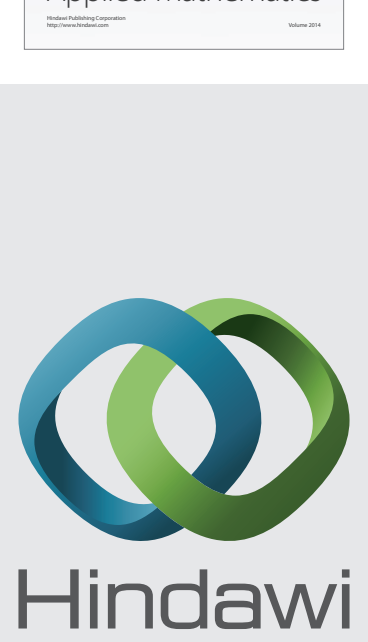

Submit your manuscripts at http://www.hindawi.com
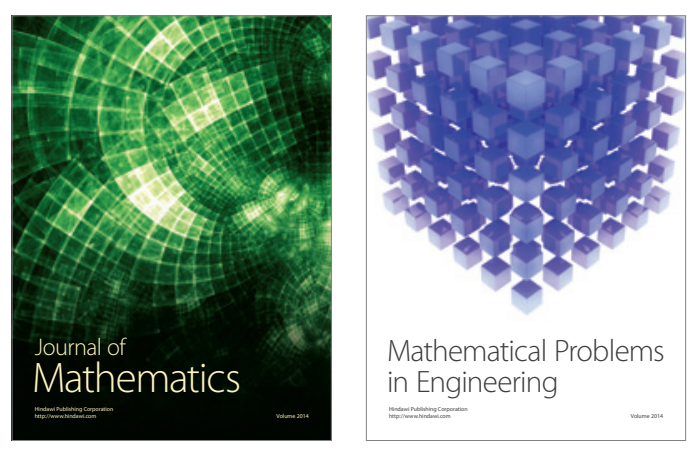

Mathematical Problems in Engineering
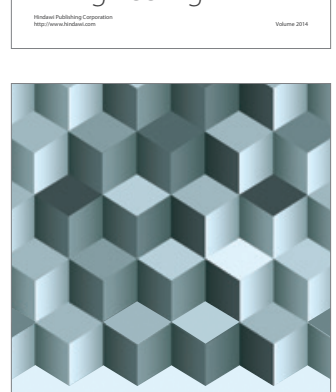

Journal of

Function Spaces
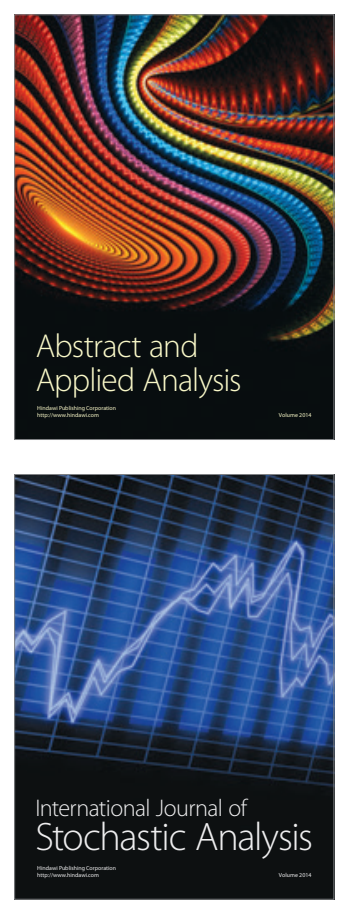

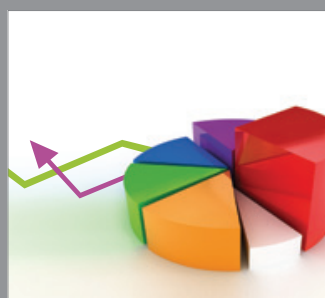

ournal of

Probability and Statistics

Promensencen
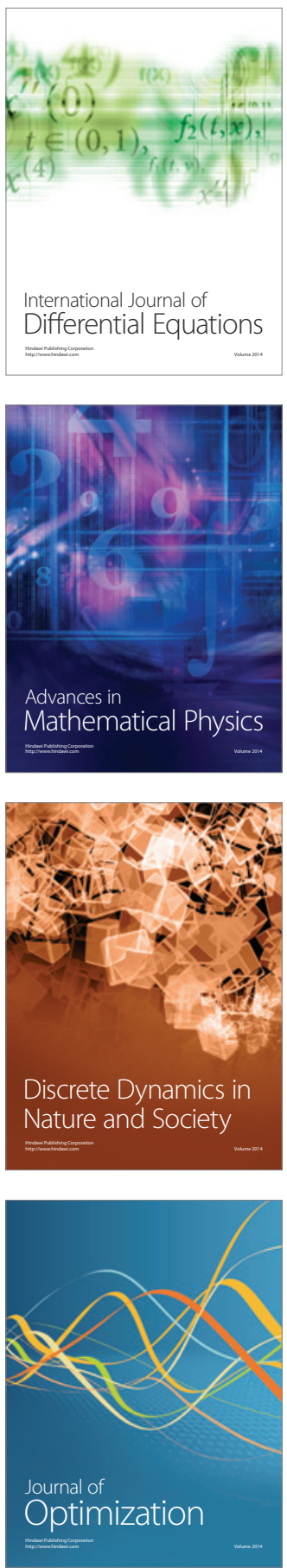